\title{
A Superconvergent Monte Carlo Method for Multiple Integrals on the Grid
}

\author{
Sofiya Ivanovska, Emanouil Atanassov, and Aneta Karaivanova \\ Institute for Parallel Processing - Bulgarian Academy of Sciences, \\ Acad. G. Bonchev St., Bl.25A, 1113 Sofia, Bulgaria \\ \{sofia, emanouil, anet\}@parallel.bas.bg
}

\begin{abstract}
In this paper we present error and performance analysis of a Monte Carlo variance reduction method for solving multidimensional integrals and integral equations. This method combines the idea of separation of the domain into small subdomains with the approach of importance sampling. The importance separation method is originally described in our previous works [7,9. Here we present a new variant of this method adding polynomial interpolation in subdomains. We also discuss the performance of the algorithms in comparison with crude Monte Carlo. We propose efficient parallel implementation of the importance separation method for a grid environment and we demonstrate numerical experiments on a heterogeneous grid. Two versions of the algorithm are compared - a Monte Carlo version using pseudorandom numbers and a quasi-Monte Carlo version using the Sobol and Halton low-discrepancy sequences 13,8 .
\end{abstract}

\section{Introduction}

Consider the problem of approximate calculation of the multiple integral

$$
I[f]=\int_{G} f(x) p(x) d x, \quad p(x) \geq 0, \quad \int_{G} p(x) d x=1,
$$

where $p(x)$ is a probability density function. The crude Monte Carlo quadrature is based on the probability interpretation of the integral:

$$
I_{N}[f]=\frac{1}{N} \sum_{n=1}^{N} f\left(x_{n}\right),
$$

where $\left\{x_{n}\right\}$ is distributed according to $p(x)$. The error is proportional to $\sigma[f] N^{-\frac{1}{2}}$ :

$$
\epsilon_{N}[f] \sim \sigma[f] N^{-1 / 2},
$$

where

$$
\sigma[f]=\left(\int_{G}(f(x) p(x)-I[f])^{2} d x\right)^{1 / 2} .
$$


There are various ways to improve the convergence rate of Monte Carlo integration. The following theorem, due to Bachvalov, establishes a lower bound for both Monte Carlo and deterministic integration formulae for smooth functions:

Theorem 1. (Bachvalov [4, 5]) There exist constants $c(s, k), c^{\prime}(s, k)$, such that for every quadrature formula $I_{N}[f]$ which is fully deterministic and uses the function values at $N$ points there exists a function $f \in \mathbf{C}_{k}^{s}$ such that

$$
\left|\int_{\mathrm{E}^{s}} f(x) d x-I_{N}[f]\right| \geq c(s, k)\|f\| N^{-\frac{k}{s}}
$$

and for every quadrature formula $I_{N}[f]$, which involves random variables and uses the function values at $N$ points, there exists a function $f \in \mathbf{C}_{k}^{s}$, such that

$$
\left\{\mathrm{E}\left[\int_{\mathrm{E}^{s}} f(x) d x-I_{N}[f]\right]^{2}\right\}^{1 / 2} \geq c^{\prime}(s, k)\|f\| N^{-\frac{1}{2}-\frac{k}{s}} .
$$

From this theorem it follows that Monte Carlo methods have advantage over deterministic methods, especially in high dimensions. In order to obtain the optimal convergence rate for functions with bounded $k$-th order derivatives, one widely used technique is stratification. Split the integration region $G$ into $M$ subdomains:

$$
\begin{gathered}
G=\bigcup_{j=1}^{M} D_{j}, \quad D_{i} \cap D_{j}=\emptyset, \quad i \neq j, \quad \sum_{j=1}^{M} N_{j}=N, \\
p^{(j)}(x)=p(x) / \bar{p}_{j}, \quad \bar{p}_{j}=\int_{D_{j}} p(x) d x .
\end{gathered}
$$

In the subdomain $D_{j}$ use a Monte Carlo integration formula $I_{N_{j}}^{(j)}[f]$, which utilizes $N_{j}$ random points $\xi_{1}^{(j)}, \ldots, \xi_{N_{j}}^{(j)}$. The stratified Monte Carlo formula is

$$
I_{N}[f]=\sum_{j=1}^{M} \frac{\bar{p}_{j}}{N_{j}} I_{N_{j}}^{(j)}[f] .
$$

The error of the stratified method is given by

$$
\epsilon_{N} \sim N^{-1 / 2} \sigma_{s}, \quad \sigma_{s}^{2}=\sum_{j=1}^{M} \sigma^{(j)^{2}}, \quad \sigma^{(j)}=\left(\int_{D_{j}}\left(f(x) p_{j}(x)-I_{N_{j}}[f]\right)^{2} d x\right)^{\frac{1}{2}}
$$

In our method we achieve $\sigma^{(j)}=\mathcal{O}\left(N^{-\frac{k}{s}}\right)$, if the function $f$ is in $\mathbf{C}_{k}^{s}$, and therefore we attain the optimal convergence rate (see Sec. 2).

Another possible idea for improving the convergence of Monte Carlo methods is to replace the pseudorandom numbers with terms of a low-discrepancy 
sequence [6, 12]. The most widely used measure for estimating the quality of the distribution of a low-discrepancy sequence $\tau=\left\{x_{i}\right\}_{i=1}^{N}$ in $\mathrm{E}^{s}$ is its discrepancy

$$
D_{N}^{*}=\sup _{I \subset \mathrm{E}^{s}}\left|\frac{A_{N}(\tau, I)}{N}-\mu(\mathrm{E})\right| .
$$

Low-discrepancy sequences have order of convergence of the discrepancy $\mathcal{O}\left(N^{-1} \log ^{s} N\right)$, which becomes order of convergence of the corresponding quasiMonte Carlo method for integrating a function over $\mathrm{E}^{s}$ :

$$
Q_{N}[f]=\frac{1}{N} \sum_{j=1}^{N} f\left(x_{i}\right) .
$$

Therefore quasi-Monte Carlo methods offer higher conversion rate (at the expense of more stringent smoothness requirements). Formula 2 can be considered as a quasi-Monte Carlo version of formula 1] Designing a quasi-Monte Carlo version of a Monte Carlo method is not always straight-forward. We studied the possibility of using the Halton and Sobol low-discrepancy sequences in our algorithm in Sec. 2,

Monte Carlo methods usually show good parallel efficiency. In Sec. 3] we describe our parallelization approach, which is suitable for grid environments. We obtained good parallel efficiency in both Monte Carlo and quasi-Monte Carlo versions.

\section{Description of Our Algorithm}

We introduce adaptive division of the domain into smaller subdomains, so that smaller subdomains are used where the function values are larger. In every subdomain we approximate the function by a polynomial, using function values at some fixed points. The input parameters of our algorithm are the dimension $s$, the smoothness order $k$, the number of base points $M$, the number of steps $N$, the number of random points in each cube $m$, the number of points used for one application of crude Monte Carlo method $R$. First we select $M$ points $a_{1}, \ldots, a_{M}$ in the unit cube $\mathrm{E}^{s}$, following the procedure, discussed in [2], and compute the respective coefficients $c_{1}, \ldots, c_{M}$. For every coordinate $x_{j}$, we estimate the values of

$$
g\left(x_{j}\right)=\int_{\mathrm{E}^{s-1}}\left|f\left(x_{1}, \ldots, x_{j-1}, x_{j}, x_{j+1}, \ldots, x_{s}\right)\right| d x_{1}, \ldots, d x_{j-1} d x_{j+1}, \ldots, d x_{s}
$$

at points $x_{j}=\frac{r}{N}$, using crude Monte Carlo algorithm with $R$ points. Then we approximate the one-dimensional function $g$ by a piece-wise linear function $\tilde{g}$, using these values. For every coordinate we choose $N+1$ points $\xi_{r}^{i}$, so that $\xi_{0}^{i}=0, \xi_{N}^{i}=1$, and

$$
\int_{\xi_{r-1}^{i}}^{\xi_{r}^{i}} \tilde{g}(t) d t=\int_{\xi_{r}^{i}}^{\xi_{r+1}^{i}} \tilde{g}(t) d t, \quad r=1, \ldots, N-1 .
$$


These steps can be considered as pre-processing, and can be done in parallel, if we use more than one CPU. Using the points $\xi_{r}^{i}$, we partition the cube $\mathrm{E}^{s}$ into $N^{s}$ subdomains $D_{j}$. Approximate $\int_{D_{j}} f(x) d x$ by the formula:

$$
\mu\left(D_{j}\right) \frac{1}{m} \sum_{i=1}^{m}\left(f\left(\eta_{i}\right)-L_{j}\left(f, \eta_{i}\right)\right)+\mu\left(D_{j}\right) \sum_{i=1}^{M} c_{i} f\left(T_{j}\left(a_{i}\right)\right),
$$

where $T_{j}$ is the canonical linear transformation that maps $\mathrm{E}^{s}$ onto $D_{j}$, the points $\eta_{i} \in D_{j}$ are uniformly distributed random points, and $L_{j}\left(f, \eta_{i}\right)$ is the polynomial approximation to $f$, obtained using the values $f\left(T_{j}\left(a_{i}\right)\right)$ (see [2]). Summing these unbiased estimates, we obtain an estimate of the integral over $\mathrm{E}^{s}$. The variance of this estimate is a sum of the variances $\sigma_{j}^{2}$ in every subdomain $D_{j}$. The order of convergence is $\mathcal{O}\left(N^{-k-s / 2}\right)$, when using $\mathcal{O}\left(N^{s}\right)$ points and so it is optimal in the sense of Theorem 11. An aposteriory estimate of the error is obtained by using the empirical variances $\tilde{\sigma_{j}}$.

The number of function evaluations is $N^{s}(m+M)+R s(N-1)$. In most practical situations the time for performing them dominates in the whole computation.

We studied also quasi-Monte Carlo variants of the algorithm. In Monte Carlo methods one uses pseudorandom number generators in order to sample uniformly distributed random variables. The idea of quasi-Monte Carlo methods is to replace the pseudorandom numbers with terms of a low-discrepancy sequence. When integrating a function in the $s$-dimensional unit cube, the dimension of the low-discrepancy sequence is frequently higher than $s$, if a more complex algorithm is used. We note that when used in parallel computations, low-discrepancy sequences can offer exact reproducibility of results, unlike Monte Carlo. In our algorithm the coordinates of the $r$-th term of the sequence can be used to produce the coordinates of the points $\eta_{i}$. Since we have $s$ coordinates and $m$ points, the constructive dimension of the algorithm becomes $\mathrm{sm}$. We tested two of the most popular families of low-discrepancy sequences - the sequences of Sobol and Halton. Since the rate of convergence of the quasi-Monte Carlo algorithm has a factor of $\log ^{s m} N$, we did not observe improvement with respect of the Monte Carlo version (see Sec. 4).

\section{Parallel Implementation of the Algorithm Suitable for Computational Grids}

Monte Carlo methods are inherently parallelizable, and both coarse-grain and fine-grain parallelism can be exploited. We take into account the fact that the Grid is a potentially heterogeneous computing environment, where the user does not know the specifics of the target architecture. Therefore parallel algorithms should be able to adapt to this heterogeneity, providing automated load-balancing. Monte Carlo algorithms can be tailored to such environments, provided parallel pseudo-random number generators are available. The use of 
quasi-Monte Carlo algorithms poses more difficulties. In both cases the efficient implementation of the algorithms depends on the functionality of the corresponding packages for generating pseudorandom or quasirandom numbers.

As a package of parallel pseudo-random generators we used the SPRNG ([10]). For generating the scrambled Halton and Sobol sequences, we used our ultra-fast generators $([1,3])$, which provide the necessary functionality:

- portable

- use assembly language for best performance on various Intel Pentium and AMD processors

- provide a fast-forward operation, important for our parallelization approach.

Our parallelization is based on the master-slave paradigm, with some ramifications. We divide the work into chunks, corresponding to the subdomains, which are requested from the master process by the slave processes. In order to increase the efficiency, the master also performs computations, while waiting for communication requests. Thus we achieve overlap of computations and communications, and we do not lose the possible output of the master process. When using low-discrepancy sequences, we take care in both master and slave processes to fast-forward the generator exactly to the point that is needed. The scrambling that is provided by the generators enables aposteriory estimation of the error in the quasi-Monte Carlo case.

\section{Numerical Experiments}

Our numerical tests are based on the next two examples, which are taken from paper of Moskowitz and Caflisch [11.

Example 1. The first example is Monte Carlo integration over $E^{5}=[0,1]^{5}$ of the function

$$
f_{1}(x)=\exp \left(\sum_{i=1}^{5} a_{i} x_{i}^{2} \frac{2+\sin \left(\sum_{j=1, j \neq i}^{5} x_{j}\right)}{2}\right),
$$

where $\mathbf{a}=\left(1, \frac{1}{2}, \frac{1}{5}, \frac{1}{5}, \frac{1}{5}\right)$.

Example 2. The second example is Monte Carlo integration over $\mathrm{E}^{7}=[0,1]^{7}$ of the function

$$
\begin{gathered}
f_{2}(x)=e^{1-\left(\sin ^{2}\left(\frac{\pi}{2} x_{1}\right)+\sin ^{2}\left(\frac{\pi}{2} x_{2}\right)+\sin ^{2}\left(\frac{\pi}{2} x_{3}\right)\right)} \\
\times \arcsin \left(\sin (1)+\frac{x_{1}+\cdots+x_{7}}{200}\right) .
\end{gathered}
$$

Table 1 shows the results for 5-dimensional integral. The smoothness that is used is 4 or 6 . And just for comparison we show results with crude Monte Carlo method that uses the same number of functional values as our algorithm. In 
Table 1. Results for Example 1 with number of cubes $N$ and number of points per cube 10

\begin{tabular}{||c||c||c|c||c||c||}
\hline \hline$N$ & $\mathrm{k}$ & SPRNG & Sobol & Halton & crude MC \\
\hline \hline \multirow{2}{*}{2} & 4 & $5.15 \mathrm{e}-04$ & $3.16 \mathrm{e}-04$ & $3.72 \mathrm{e}-04$ & $1.52 \mathrm{e}-02$ \\
\cline { 2 - 5 } & 6 & $7.06 \mathrm{e}-05$ & $1.43 \mathrm{e}-05$ & $2.34 \mathrm{e}-05$ & $7.40 \mathrm{e}-03$ \\
\hline \hline \multirow{2}{*}{3} & 4 & $8.01 \mathrm{e}-05$ & $4.38 \mathrm{e}-05$ & $4.77 \mathrm{e}-05$ & $5.51 \mathrm{e}-03$ \\
\cline { 2 - 5 } & 6 & $2.15 \mathrm{e}-07$ & $7.41 \mathrm{e}-07$ & $3.19 \mathrm{e}-07$ & $2.68 \mathrm{e}-03$ \\
\hline \hline \multirow{2}{*}{4} & 4 & $1.21 \mathrm{e}-05$ & $1.01 \mathrm{e}-05$ & $2.09 \mathrm{e}-05$ & $2.68 \mathrm{e}-03$ \\
\cline { 2 - 5 } & 6 & $2.95 \mathrm{e}-07$ & $3.45 \mathrm{e}-07$ & $1.35 \mathrm{e}-07$ & $1.31 \mathrm{e}-03$ \\
\hline \hline \multirow{2}{*}{5} & 4 & $1.78 \mathrm{e}-06$ & $3.03 \mathrm{e}-06$ & $2.87 \mathrm{e}-06$ & $1.54 \mathrm{e}-03$ \\
\cline { 2 - 6 } & 6 & $2.79 \mathrm{e}-08$ & $3.61 \mathrm{e}-08$ & $4.09 \mathrm{e}-08$ & $7.48 \mathrm{e}-04$ \\
\hline \hline
\end{tabular}

Table 2. Results for Example 2 with number of cubes $N$ and number of points per cube 10

\begin{tabular}{|c|c|c|c|c|}
\hline & & & $\overline{\text { Sobol }}$ & \\
\hline & & $.25 \mathrm{e}-05$ & $\overline{.12}$ & \\
\hline & & & 7 & 78 \\
\hline \multirow[t]{2}{*}{ J } & & 6 & .30 & 4.6 \\
\hline & & 7.57 & $7.61 \mathrm{e}-08$ & 8.61 \\
\hline \multirow[t]{2}{*}{4} & & 2.94 & $1.82 \mathrm{e}-06$ & 4.7 \\
\hline & & 5.99 & $1.07 \mathrm{e}-08$ & $1.44 \mathrm{e}-0$ \\
\hline \multirow[t]{2}{*}{5} & & 1.2 & $3.21 \epsilon$ & $.43 \mathrm{e}$ \\
\hline & & & $c_{1}$ & \\
\hline
\end{tabular}

both examples the errors obey the theoretical laws, i.e., the convergence rate is $\mathcal{O}\left(N^{-\frac{1}{2}}\right)$ for the crude Monte Carlo method, and $\mathcal{O}\left(N^{-\frac{k}{s}-\frac{1}{2}}\right)$ for our adaptive method, when smoothness $k$ is used. Table 3 shows CPU time for our algorithm when $N$ is equal to 14 and the number of random points per cube $m$ is equal to 40. We used different computers for these tests - three of the computers have Pentium processors running at different clock speeds and one has a Motorola G4 processor. All of the computers had the Globus toolkit installed. In Table 4 we compare the estimated time for running our algorithm on all those computers in parallel, in case of perfect parallel efficiency, with the measured execution time. We used the MPICH-G2 implementation of MPI, which is the most general approach for running parallel jobs on computational grids. In this way we successfully utilized machines with different endianness in the same computation. We obtained roughly the same accuracy with low-discrepancy sequences as with pseudo-random numbers, due to the high effective dimension. The CPU time of our implementations of the quasi-Monte Carlo versions was frequently smaller, due to the efficient generation algorithms. Quasi-Monte Carlo algorithms are more difficult to parallelize under such constraints, but this can be done if the generators of the low-discrepancy sequences have the necessary functionality. 
Table 3. Time and efficiency for Example 1 with number of points 14 and number of points per cube 40

\begin{tabular}{||c|c|c|c|c||}
\hline \hline & $\mathrm{P} 4 / 2.8 \mathrm{GHz}$ & $\mathrm{P} 4 / 2 \mathrm{GHz}$ & $\mathrm{P} 4 / 2 \mathrm{GHz}$ & $\mathrm{G} 4 / 450 \mathrm{MHz}$ \\
\hline \hline SPRNG & 102 & 117 & 118 & 413 \\
\hline Sobol & 91 & 106 & 96 & 393 \\
\hline Halton & 91 & 106 & 106 & 393 \\
\hline \hline
\end{tabular}

Table 4. Parallel efficiency measurements

\begin{tabular}{||c|c|c|c||}
\hline \hline & Estimated Time & Measured Time & Efficiency \\
\hline \hline SPRNG & 34 & 41 & $83 \%$ \\
\hline Sobol & 30 & 34 & $88 \%$ \\
\hline Halton & 31 & 34 & $91 \%$ \\
\hline \hline
\end{tabular}

\section{Conclusions}

An adaptive Monte Carlo method for solving multiple integrals of smooth functions has been proposed and tested. This method is an improved version of the importance separation method. The importance separation method is combined with polynomial interpolation in the subdomains. A quasi-Monte Carlo version of the algorithm was also studied. The obtained results are not an efficient parallel implementation of the algorithm has been achieved using a version of the master-slave computing paradigm, enabling the execution of the programs in heterogeneous Grid environments. The ideas of this implementation can be extended to other Monte Carlo and quasi-Monte methods.

Acknowledgments. This work is supported by the Ministry of Education and Science of Bulgaria under Grant \# I-1405/04.

\section{References}

1. E. Atanassov. Measuring the Performance of a Power PC Cluster, Computational Science - ICCS 2002 ( P. Sloot, C. Kenneth Tan, J. Dongarra, A. Hoekstra - Eds.), LNCS 2330, 628-634, Springer, 2002.

2. E. Atanassov, I. Dimov, M. Durchova. A New Quasi-Monte Carlo Algorithm for Numerical Integration of Smooth Functions, Large-Scale Scientific Computing (I. Lirkov, S. Margenov, J. Wasniewski, P. Yalamov - Eds.), LNCS 2907, 128-135, Springer, 2004.

3. E. Atanassov, M. Durchova. Generating and Testing the Modified Halton Sequences, Numerical Methods and Applications (I. Dimov, I. Lirkov, S. Margenov, Z. Zlatev - Eds.), LNCS 2542, 91-98, Springer, 2003.

4. N.S. Bachvalov. On the approximate computation of multiple integrals, Vestnik Moscow State University, Ser. Mat., Mech., Vol. 4, 3-18, 1959. 
5. N.S. Bachvalov. Average Estimation of the Remainder Term of Quadrature Formulas, USSR Comput. Math. and Math. Phys., Vol. 1(1), 64-77, 1961.

6. R.E. Caflisch. Monte Carlo and quasi-Monte Carlo methods, Acta Numerica, Vol. 7, 1-49, 1998.

7. I. Dimov, A. Karaivanova, R. Georgieva and S. Ivanovska. Parallel Importance Separation and Adaptive Monte Carlo Algorithms for Multiple Integrals, Numerical Methods and Applications (I. Dimov, I. Lirkov, S. Margenov, Z. Zlatev - Eds.), LNCS 2542, 99-107, Springer, 2003.

8. J. H. Halton. On the efficiency of certain quasi-random sequences of points in evaluating multi-dimensional integrals, Numer. math., 2, 84-90, 1960.

9. A. Karaivanova. Adaptive Monte Carlo methods for numerical integration, Mathematica Balkanica, Vol. 11, 391-406, 1997.

10. M. Mascagni. SPRNG: A Scalable Library for Pseudorandom Number Generation. Recent Advances in Numerical Methods and Applications II (O. Iliev, M. Kaschiev, Bl. Sendov, P.S. Vassilevski eds.), Proceeding of NMA 1998, World Scientific, Singapore, 284-295, 1999.

11. B. Moskowitz and R.E. Caflisch. Smoothness and dimension reduction in quasiMonte Carlo methods, J. Math. Comput. Modeling, 23, 37-54, 1996.

12. H. Niederreiter. Random number generation and quasi-Monte Carlo methods, SIAM, Philadelphia, 1992.

13. I.M. Sobol. On the distribution of point in a cube and the approximate evaluation of integrals, USSR Computational Mathematics and Mathematical Physics, 7, 86112, 1967. 